\title{
TIME-DEPENDENT TRANSPORT THROUGH A QUANTUM DOT: MEAN-FIELD TREATMENT OF THE COULOMB INTERACTION
}

T. Kwapiński, R. Taranko and E. Taranko

Institute of Physics, M. Curie-Skłodowska University

Pl. M. Curie-Skłodowskiej 1, 20-031 Lublin, Poland

(Received August 1, 2000; revised version December 12, 2000)

We study an electron transport through a quantum dot in the presence of time-dependent fields. The dot is described by a single level and the intra-dot Coulomb interaction is introduced within the Hartree-Fock approximation. The external fields cause a time-dependent shift of the energy spectrum of the leads and quantum dot. We take into account the spin-polarized solutions for the quantum dot charge. We calculated the time dependence of the current and charge accumulated on the dot, the average current and charge vs. the source-drain and gate voltages in dependence on the Coulomb interaction strength and the frequencies and amplitude of the external fields.

PACS numbers: $85.30 . \mathrm{Vw}, 85.30 . \mathrm{Wx}$

\section{Introduction}

The advance of experimental techniques on a nanometer scale has made it possible to investigate the electronic transport through quantum dots - small structures formed in two-dimensional electron gas by applying appropriate gate voltage. Especially interesting are the transport properties of a quantum dot (QD) under the influence of external time-dependent fields. The high-frequency signals may be applied to the QD and the time-dependent fields will modify the tunneling current. We should mention such phenomena as a photon-assisted tunneling (e.g. [1]) or photon-electron pump effect, turnstiles and others (e.g. [2]). One can investigate the current flowing through the QD under the periodic modulation of the QD electronic structure [3] or periodic modulation of the tunneling barriers [2] and electron energy levels in both (left and right) reservoirs [4] (see also [5-8]). The effect of the time-dependent potential on the conductance and current flowing through the QD and superlattices has been measured and some interesting features, e.g. negative conductance, have been observed $[1,3,9]$. 
The Coulomb intra-dot electron-electron interactions play very important role in some phenomena, such as the Coulomb blockade oscillations, resonant tunneling, and many others. It is well known that the Coulomb interactions make the tunneling coefficient very small in the blockade regime in opposite to the tunneling regime where the current flowing through the quantum dot is measured. The conductance exhibits oscillations as a function of the external potential which can be explained by the mechanism of single-electron tunneling and Coulomb blockade phenomenon due to intra-dot electron-electron interaction.

In the literature there are numerous theoretical works concerning the influence of the intra-dot electron-electron interaction in time-independent electron transport through the quantum dot (e.g. [10-14]). On the other hand, the literature on time-dependent nonequilibrium transport treated together with the Coulomb interactions is much more restricted in comparison with the stationary case. In Refs. [8, 10] the transport through a QD in the presence of time-dependent microwaves was studied. The QD was characterized by two single electronic levels and the intra-dot Coulomb interaction. The QD retarded Green function for finite values of the Coulomb interactions was calculated using the equation of motion method and the time-dependent and average current was obtained. On the other hand, the nonlinear tunneling current through the QD in the Kondo regime assuming the infinite $U$ value was calculated in Ref. [10].

The Coulomb interaction in many small quantum structures is small compared with the energy difference between non-interacting eigenstates of the QD structure. For this reason, a mean-field model should give, for many purposes, a relatively good description of the system. Sun and Lin [7] used the Hartree-Fock approximation in description of the electron tunneling through the QD and calculated the time-dependent current and the average current as a function of gate voltage. The corresponding equations needed to be solved are very complicated and the authors make the additional approximations (described later) in their numerical approach. It seems, however, that such kind of approximations can influence the results, especially, if one considers the time-dependent characteristics. In this paper we calculate the time-dependent current flowing through the QD, the charge accumulated on the dot, the average current vs. the gate voltage or source-drain voltage and the conductance for the model in which the Coulomb interaction is taken in a mean-field manner without any additional approximations as it was made in Ref. [7]. We present a simple method according to which we are able to solve the self-consistent time-dependent equations for the charge accumulated on the QD without doing any approximations.

The outline of this paper is as follows. In Sec. 2 the model is given and the basic ideas underlying the method are presented. In Sec. 3 we give the numerical results for currents, QD charge, and conductances obtained for different electronic characteristics of the system and different time-dependent microwave fields. Section 3 contains also conclusions and a brief summary. 


\section{Hamiltonian and formalism}

Our mesoscopic system is composed of three parts: the left metal lead, the central quantum dot, and the right lead. The three separate parts are coupled together through the tunneling barriers. The chemical potentials $\mu_{\alpha}(\alpha=\mathrm{L}, \mathrm{R})$ of the two metal leads may be not equal, and their difference is not necessarily small. The Hamiltonian of the system can be written as follows:

$$
\begin{aligned}
H= & \sum_{\mathbb{R} \sigma, \alpha} \varepsilon_{\boldsymbol{k} \alpha}(t) a_{\boldsymbol{k} \alpha \sigma}^{+} a_{\boldsymbol{R} \alpha \sigma}+\sum_{\mathbb{k} \sigma, \alpha}\left(V_{\boldsymbol{k} \alpha} a_{\boldsymbol{k} \alpha \sigma}^{+} a_{d \sigma}+\text { h.c. }\right) \\
& +\frac{U}{2} \sum_{\sigma} n_{d-\sigma} n_{d \sigma}+\sum_{\sigma} \varepsilon_{d}(t) a_{d \sigma}^{+} a_{d \sigma},
\end{aligned}
$$

where $\alpha$ denotes the left (L) or right ( $\mathrm{R})$ lead. The operators $a_{\boldsymbol{k} \alpha \sigma}\left(a_{\boldsymbol{k} \alpha \sigma}^{+}\right), a_{d \sigma}\left(a_{d \sigma}^{+}\right)$ are the annihilation (creation) operators of the electron in the leads (right and left) and in the dot, respectively. For simplicity the dot is characterized only by a single level $\varepsilon_{d}$ and the intra-dot Coulomb electron-electron interaction $U$ and $\varepsilon_{\alpha}$ denotes the single-electron energies of the left or right lead. The transfer-matrix elements between the leads and the dot are denoted by $V_{\boldsymbol{k} \alpha}$.

We consider the case in which there exist microwave fields applied to the leads and to the quantum dot. We have taken the adiabatic approximation in our time-dependent driven system so that the fields can be reflected in the single-electron energies as follows:

$$
\varepsilon_{\text {k } \alpha}(t)=\varepsilon_{k \alpha}+\Delta_{\alpha} \cos \omega t, \quad \varepsilon_{d}(t)=\varepsilon_{d}+\Delta_{d} \cos \omega t,
$$

i.e. the energy levels of the leads and QD are driven by the ac field with frequency $\omega$ and amplitudes $\Delta_{\alpha}$ and $\Delta_{d}$, respectively. However, the time-dependent external field causes only a rigid shift of the energy levels and does not change the distribution of occupation of electrons [5, 7]. The amplitudes, phases, and frequencies of the external fields can be taken arbitrarily in different regions of our system.

The general formulas for the time-dependent current flowing through the QD and for the charge accumulated on the QD can be obtained using the non-equilibrium Green function technique [5, 7,12$]$. These general formulas contain, among others, the retarded, two-time Green function of the dot connected with left and right leads. The exact results for it is not known even in the time-independent case, then the approximations are needed. One can use, for example, the equation of motion method which with some decoupling procedures can lead to final approximate results (e.g. [7]). If these decouplings are used on the early stage of the equation of motion scheme then one recovers the mean field result, namely, the Hartree-Fock approximation. On the other hand, one can use from the very beginning the mean-field Hamiltonian, in which, in comparison with Eq. (1), the last two terms are replaced with $\sum_{\sigma}\left[\varepsilon_{d}(t)+U N_{d-\sigma}(t)\right] a_{d \sigma}^{+} a_{d \sigma}$, where $N_{d \sigma}(t)$ is the time-dependent charge localized on the dot. Now, the calculations of the required characteristics of the system under investigations can be obtained similarly as in the non-interacting (without electron correlations) case. Without describing the formalism, which has already been discussed in detail (see, e.g. [5-8, 12]), we recall 
that the current flowing from the left lead into the QD and the charge accumulated on the QD, $N_{d \sigma}(t)$, can be expressed as

$$
\begin{aligned}
& I_{\mathrm{L}}(t)=\sum_{\sigma}\left[-\Gamma^{\mathrm{L}} N_{d \sigma}(t)-\frac{\Gamma^{\mathrm{L}}}{\pi} \int \mathrm{d} \varepsilon f_{\mathrm{L}}(\varepsilon) \operatorname{Im} A_{\mathrm{L}}^{\sigma}(\varepsilon, t)\right], \\
& N_{d \sigma}(t)=\int \frac{\mathrm{d} \varepsilon}{2 \pi} f_{\mathrm{L}}(\varepsilon) \Gamma^{\mathrm{L}}\left|A_{\mathrm{L}}^{\sigma}(\varepsilon, t)\right|^{2},
\end{aligned}
$$

where

$$
\begin{gathered}
A_{\mathrm{L}}^{\sigma}(\varepsilon, t)=-\mathrm{i} \exp \left[\mathrm{i}\left(\Delta_{\alpha}-\Delta_{d}\right) \sin \omega t / \omega\right] \int_{-\infty}^{t} \mathrm{~d} t_{1} \exp \left[\mathrm{i}\left(\varepsilon-\varepsilon_{d}\right)\left(t-t_{1}\right)\right. \\
\left.-\Gamma\left(t-t_{1}\right) / 2-\mathrm{i}\left(\Delta_{\alpha}-\Delta_{d}\right) \sin \omega t_{1} / \omega-\mathrm{i} \int_{t_{1}}^{t} \mathrm{~d} t^{\prime \prime} U N_{d-\sigma}\left(t^{\prime \prime}\right)\right]
\end{gathered}
$$

$\Gamma=\Gamma^{\mathrm{R}}+\Gamma^{\mathrm{L}}, f_{\alpha}(\varepsilon)$ denotes the Fermi distribution function of the $\alpha$-th lead and $\Gamma^{\alpha}(\varepsilon)=\Gamma^{\alpha}=2 \pi \sum_{\mathbb{R}_{k}}\left|V_{\boldsymbol{k} \alpha}\right|^{2} \delta\left(\varepsilon-\varepsilon_{\boldsymbol{k} \alpha}\right)$.

The formulas (3)-(5) were obtained within, so-called, wide-band approximation consisting of neglecting the level shift $\Lambda(\varepsilon)$ and assuming the energy independent line width $\Gamma(\varepsilon)=\Gamma$, where $\Lambda(\varepsilon)-\mathrm{i} \Gamma(\varepsilon)=\sum_{\boldsymbol{k}, \alpha}\left|V_{\boldsymbol{k} \alpha}\right|^{2} /\left(\varepsilon^{+}-\varepsilon_{\alpha}\right)$.

For calculations we take $e=\hbar=1$ units, the time is measured in units of $\hbar / \Gamma$, the current and conductance in units of $e \Gamma / \hbar$ and $e^{2} \Gamma / \hbar$ respectively, the modulation frequency $\omega$ in units of $\Gamma / \hbar$, the QD charge in units of $e$, and all the energies in units of $\Gamma$. In the following we assume $\Gamma^{\mathrm{L}}=\Gamma^{\mathrm{R}}=\Gamma / 2$ (symmetrical barriers).

Note that in order to obtain the current $I_{\mathrm{L}}(t)$ or charge accumulated on the dot one has to solve in a self-consistent manner Eqs. (4) and (5). If we neglect the Coulomb electron-electron interaction, then the function $A_{\mathrm{L}}^{\sigma}(\varepsilon, t)$ can be transformed to the simple expression consisting of the sum of the Bessel functions multiplied by some energy and time dependent factors [5]. As a result, the current $I_{\mathrm{L}}(t)$ and $\mathrm{QD}$ charge can be obtained by simple (numerical) integration as indicated in Eqs. (3), (4).

However, for non-vanishing Coulomb interaction, one has to solve the set of self-consistent Eqs. (4), (5). Note that the number of these nonlinear equations is quite large and depends on the numerical integration method used in computation of $N_{d \sigma}(t)$. Therefore, in the literature this problem was solved in an approximate manner, namely, the function $N_{d-\sigma}(t)$ in the right-hand side of Eq. (5) was replaced with a some constant mean value $\left\langle N_{d-\sigma}(t)\right\rangle[7]$. Note, however, that for time-dependent external perturbations the dot charge has to be obtained in a dynamical manner as its value at some time, say $t_{1}$, should depend on its values at the time earlier than $t_{1}$. Then, the approximation consisting of replacement of the full time-dependent dot charge in Eq. (5) by its mean value can change the time-dependent characteristics of the electron tunneling processes through the QD. Our results presented in the next section support these assumptions.

To solve the set of Eqs. (4), (5) without any additional approximations we propose the following procedure: Eq. (5) should be replaced with the differential equation

$$
\frac{\partial A_{\alpha}^{\sigma}(\varepsilon, t)}{\partial t}=\mathrm{i} A_{\alpha}^{\sigma}(\varepsilon, t)\left[\left(\Delta_{\alpha}-\Delta_{d}\right) \cos \omega t+\varepsilon-\varepsilon_{d}+\mathrm{i} \Gamma / 2-U N_{d-\sigma}(t)\right]-\mathrm{i}
$$


Equation (6) is equivalent to Eq. (5) as one can check writing its formal solution. Inserting $N_{d \sigma}(t)$, Eq. (4), to Eq. (6) one obtains the set of differential equations which can be solved by numerical integration. The number of Eqs. (6) we have to solve simultaneously depends on the numerical integration method used for calculations of the dot charge, Eq. (4), and is of order 1000-2000. Note that in order to calculate the dot charge at time $t$ we need the information about the dot charge for all time points earlier than $t$.

From numerical point of view, the set of differential Eqs. (6) is very simple to solve in comparison with the troublesome set of Eqs. (4), (5). As our method of calculations is relatively simple (and fast, from numerical calculations point of view) then we are able to consider the tunneling current, the dot charge (the time-dependent values and average values), as well as other characteristics, e.g. the differential conductance vs. source-drain voltage, for broad class of parameters of the considered system. The results of such calculations are presented in the next section.

\section{Results and discussion}

We present the results for the current flowing between the left lead and the $\mathrm{QD}$ and the charge accumulated on the QD vs. time for various values of the correlation strength $U$, the modulation frequencies $\omega$, and amplitudes $\Delta_{\mathrm{L} / \mathrm{R}}, \Delta_{d}$ of the energy levels modulation. We present also results for the current, averaged current, and for differential conductance vs. source-drain voltage and gate voltage.

We assume the temperature $T=0 \mathrm{~K}$ and the amplitude of oscillations $\Delta_{d}$ to be one half of $\Delta_{\mathrm{L}}$ and $\Delta_{\mathrm{R}}=0$. The time-average of a time-dependent current is defined by

$$
\langle I(t)\rangle=\lim _{2 \tau \rightarrow \infty} \frac{1}{2 \tau} \int_{-\tau}^{\tau} \mathrm{d} t^{\prime} I\left(t^{\prime}\right)
$$

and because $I(t)$ is aperiodic function of time, we average it over the period $2 \pi / \omega$.

Without loss of generality we take the chemical potential of the right lead $\mu_{\mathrm{R}}$ as the energy measurement reference point, $\mu_{\mathrm{R}}=0$. The potential drop between the left and right leads is given by $\mu_{\mathrm{L}}-\mu_{\mathrm{R}}=\mathrm{eV}$ and $V$ is the measured voltage between source and drain. Our calculations are performed with parameters in the range of the experiments already available, e.g. [15-17].

To find the solutions of Eqs. (4) and (6) we must first consider the following problem. Solving Eqs. (4), (6) as they stand, we obtain only paramagnetic solutions, $N_{d \sigma}(t)=N_{d-\sigma}(t)$ and the ferromagnetic solutions (if any) are missing. The similar problem is known in the theory of the charge transfer in atom-surface collisions [18]. The solutions $N_{d \sigma}$ of the time-dependent Anderson model never go to the spin-polarized ones, even for parameters which correspond, in a static case, to a strong magnetic case. As the spin-polarized solutions of the Anderson model (if only exist) are energetically more stable (e.g. the chemisorption case [18]) than for correct description of the QD one should take into consideration the ferromagnetic occupation numbers $N_{\uparrow}(t)$ and $N_{\downarrow}(t)$, rather than paramagnetic ones. Therefore, in order to obtain the spin-polarized solutions we introduce an asymme- 
try in spin space assuming that the quantum dot's energy level is spin-dependent $\varepsilon_{d} \rightarrow \varepsilon_{d}-\sigma h$, where $h$ is a small constant value and $\sigma= \pm 1$ (cf. [19,20]). As a result, the spin-polarized solutions can be obtained. In Fig. 1 we show the time dependence of the dot charge for three parameters $h$ equal to $0.0001,0.005$, and 0.05 in units of $\Gamma$, respectively. The results clearly indicate that for sufficiently long time the spin-polarized solutions are independent of $h$. As we are interested only in asymptotic behavior of the system and it is assumed that the external perturbation was adiabatically added at $t=-\infty$, then the expounded procedure of the artificial spin symmetry breaking works sufficiently good. In addition, the positions of peaks of the average current curve vs. gate voltage nearly do not depend on the spin symmetry breaking parameter $h$ (not shown here).

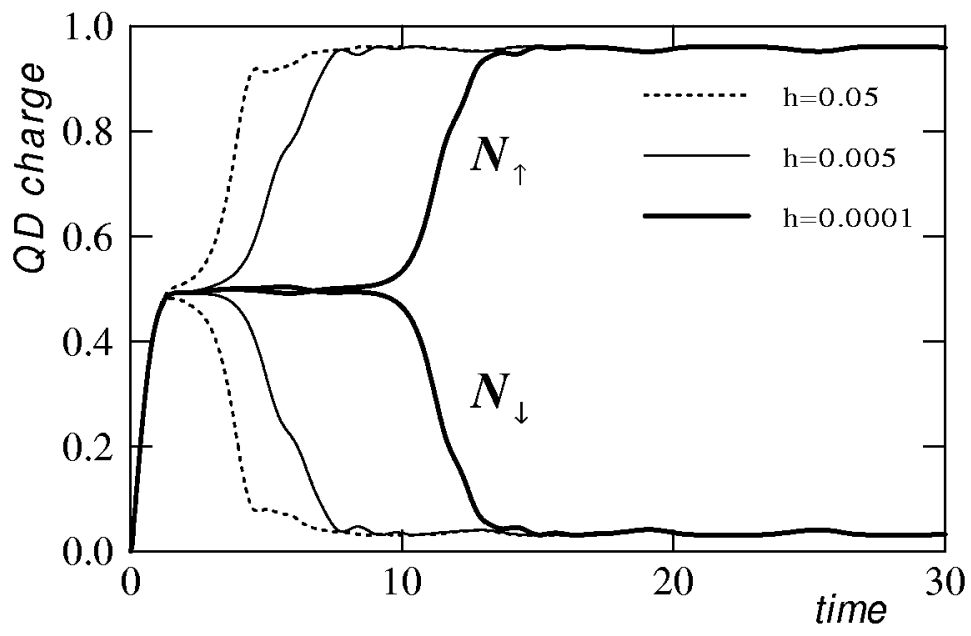

Fig. 1. The time dependence of the quantum dot charge for different values of the spin symmetry breaking parameter $h$. The thick, thin, and broken lines correspond to $h=0.0001,0.005$, and 0.05 , respectively. The unit for the time axis is $\hbar / \Gamma$, and all other energies are in units of $\Gamma: \mu_{\mathrm{L}}=2, \Delta_{\mathrm{L}}=2, \Delta_{d}=1, \varepsilon_{d}=-4, U=10$, and $\omega=1$.

In Fig. 2 we show the time dependence of the current for electron correlations $U=5,10$, and 15 for different frequencies $\omega=0.3,0.5$, and 1.0 (parts from up to down). The current and also the electric charge accumulated on the QD (Fig. 3) are periodic functions of time with the period $2 \pi / \omega$. For increasing correlation strength $U$ (with other parameters being constant) we observe that the amplitude of the current oscillations are smaller and smaller independently of the frequency $\omega$. The filling of the effective QD energy levels is different for different correlation strengths $U$ (Fig. 3). For large $U$ the solutions for $N_{\sigma}(t)$ are strongly ferromagnetic but as $U$ decreases the difference between spin-up and spin-down QD charge is smaller and smaller and tends to a paramagnetic case.

In Fig. 4 we present results for the QD charge, the average current, and the differential conductance vs. the source-drain voltage, $\mu_{\mathrm{L}}-\mu_{\mathrm{R}}$, for two values of the correlation strength $U=8$ (thin lines) and $U=12$ (thick lines). The left 


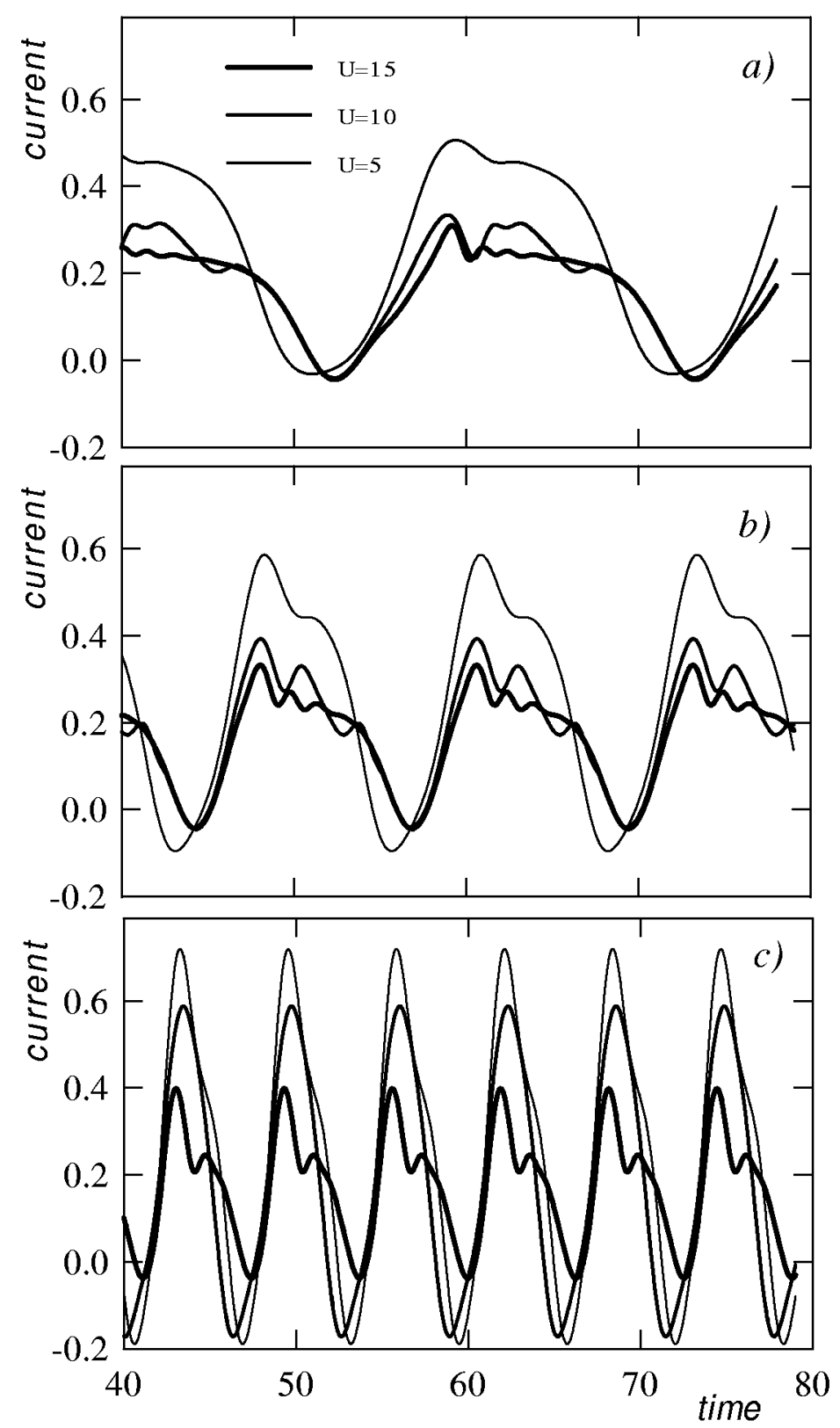

Fig. 2. The time-dependent current for $U=5,10$, and 15 (see inset in part (a)) for different modulation frequencies $\omega=0.3,0.5$, and $\omega=1-\operatorname{parts}(\mathrm{a})$, (b), and (c), respectively. $\Delta_{\mathrm{L}}=5, \Delta_{d}=2.5, \mu_{\mathrm{L}}=5$, and $\varepsilon_{d}=3$.

parts correspond to the stationary case $(\omega=0)$ and the right parts correspond to the case in which the left lead is characterized by the oscillating energy levels with amplitude $\Delta_{\mathrm{L}}=2$ and frequency $\omega=0.5$. The QD energy level $\varepsilon_{d}=2$ also 


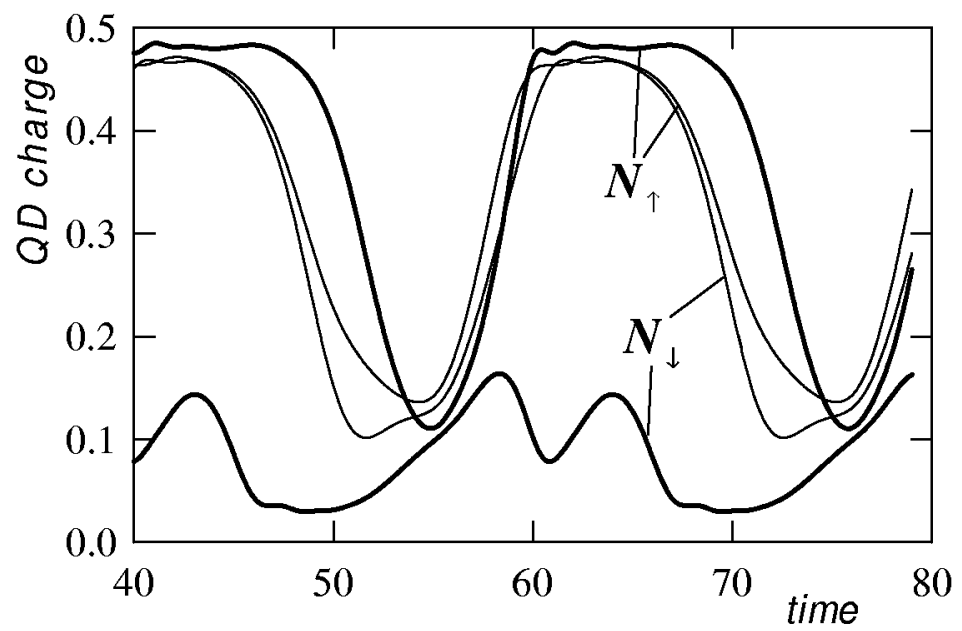

Fig. 3. The quantum dot charge for the parameters used in Fig. 2a for $U=5$ (thin lines) and $U=10$ (thick lines).

oscillates with amplitude $\Delta_{d}=1$. We observe the spin-polarized solutions only for one region of the source-drain voltage values for the stationary case $(\omega=0)$, as well as for time-dependent case $(\omega \neq 0)$. The ferromagnetic solutions exist for the case in which one of the effective QD levels, $\varepsilon_{d}+U\left\langle N_{\uparrow}\right\rangle$ or $\varepsilon_{d}+U\left\langle N_{\downarrow}\right\rangle$ is localized between $\mu_{\mathrm{L}}$ and $\mu_{\mathrm{R}}$. In all other situations there are only paramagnetic solutions.

The region of ferromagnetic solutions increases with increasing $U$ and is limited by the values of the voltage for which both effective QD levels are localized below $\min \left(\mu_{\mathrm{L}}, \mu_{\mathrm{R}}\right)$ or above $\max \left(\mu_{\mathrm{L}}, \mu_{\mathrm{R}}\right)$. The average current vs. source-drain voltage possesses two steps which appear when the effective QD levels enter into the energy region between $\mu_{\mathrm{L}}$ and $\mu_{\mathrm{R}}$. Note, however, that in the time-dependent case these steps are less clear and are characterized by the additional small structures. From comparison of the average charge with the average current we can observe that the positions (on the voltage axis) of the abrupt changing of the current coincides with the corresponding points on the charge curves. The spin-polarized QD charge results in conductance showing two peaks instead one peak as it would be for a paramagnetic case. The peaks of the conductance appear in the points where the current is changed. The structure of the conductance curve contains more details in the time-dependent case and corresponds to much more complicated structure of the current curve.

In general, the current is a complicated function of many parameters, among others, of the amplitude of energy levels oscillations $\Delta_{\mathrm{L}}$ and $\Delta_{d}$. In order to give some overall insight into this dependence we present in Fig. 5 the current as a function of the amplitude of oscillations and time. The other parameters were fixed, ie. $U=8, \omega=0.5, \varepsilon_{d}=-4, \mu_{\mathrm{L}}=2$. One can conclude that the amplitude of the current oscillations increases with increasing $\Delta_{\mathrm{L}}$ and $\Delta_{d}$. For $\Delta_{\mathrm{L}}=\Delta_{d}=0$ (absence of the energy level oscillations) the current does not flow because the QD levels are outside the energy range $\left(\mu_{\mathrm{L}}, \mu_{\mathrm{R}}\right)$. 


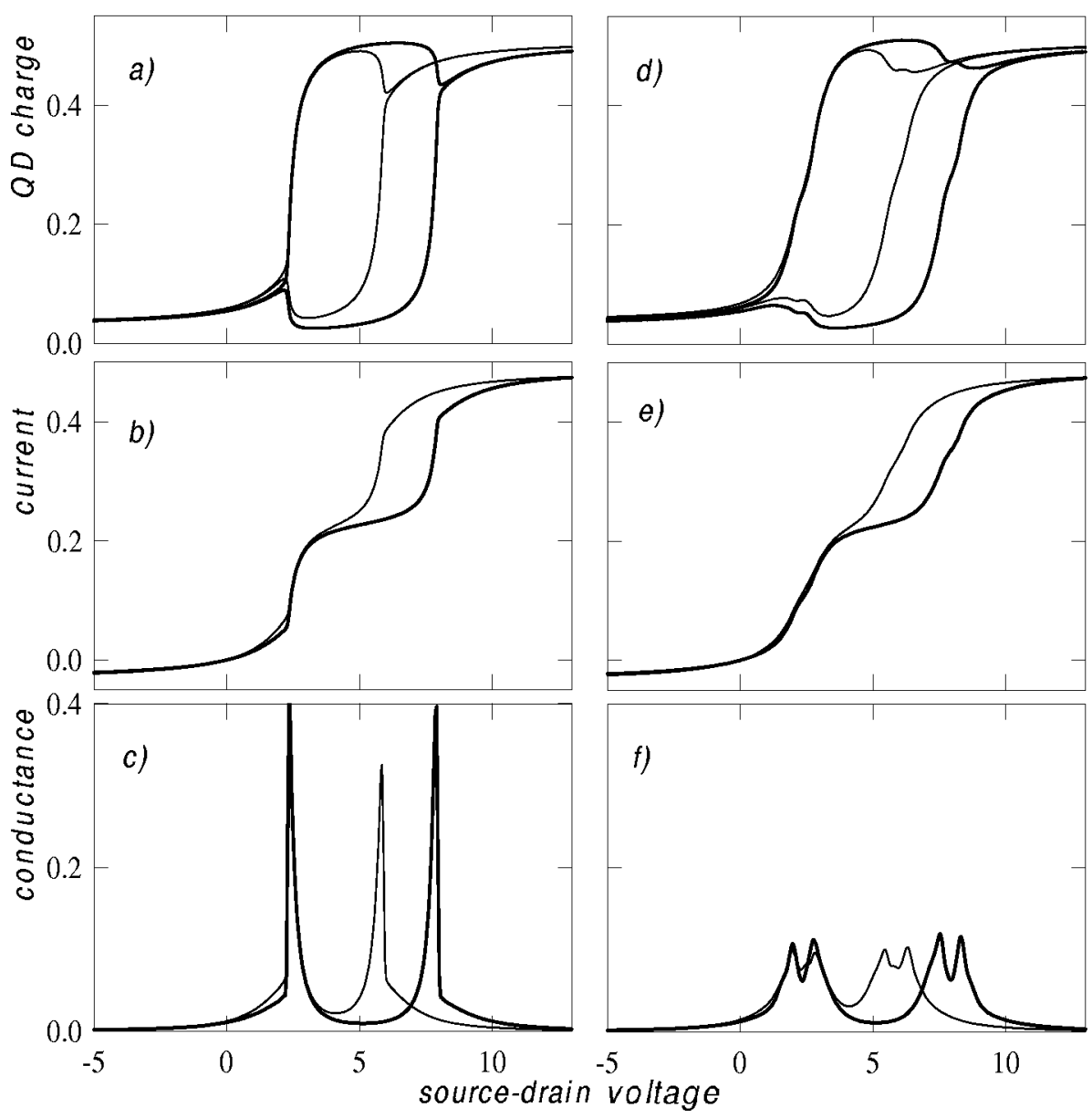

Fig. 4. The average quantum dot charge, current, and conductance vs. source-drain voltage (in $\Gamma$ units) for the static case (parts (a), (b), (c)) and for harmonic modulation case $\omega=0.5$ (parts (d), (e), (f)), $\Delta_{\mathrm{L}}=2, \Delta_{d}=1$. The thin (thick) curves correspond to $U=8(U=12)$.

In Fig. 6 we show the time- and $U$-dependence of the current $I_{\mathrm{L}}$ for the parameters: $\Delta_{\mathrm{L}}=2, \Delta_{d}=1, \mu_{\mathrm{L}}=2, \varepsilon_{d}=-4$, and $\omega=0.5$. The greatest variations of the current are observed for $4<U<7$ for which one of the effective QD levels lies between the chemical potentials $\mu_{\mathrm{L}}$ and $\mu_{\mathrm{R}}$.

The interesting results are obtained for the so-called Coulomb blockade effect. It is known that in the time-independent case for small source-drain voltages the curve current vs. gate voltage curve possesses the characteristic peaks. Only for some values of the gate voltage the current flows through the QD (see Fig. 7a). When the external time-dependent fields are applied to the system the Coulomb blockade effect is not so distinct (see Fig. 7b for $\omega=0.1$ and Fig. $7 \mathrm{c}$ for $\omega=0.5$ ). The right panels of Fig. 7 show the average charge vs. gate voltage corresponding 


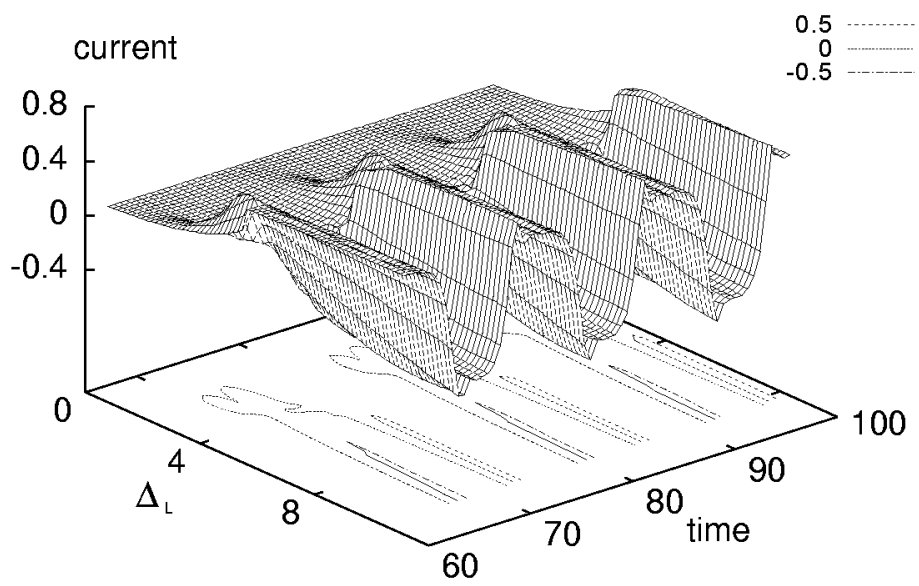

Fig. 5. The current as a function of time and $\Delta_{\mathrm{L}}$ for harmonic modulation with $\omega=0.5, \varepsilon_{d}=-4, \Delta_{d}=\Delta_{\mathrm{L}} / 2, \mu_{\mathrm{L}}=2$, and $U=8$.

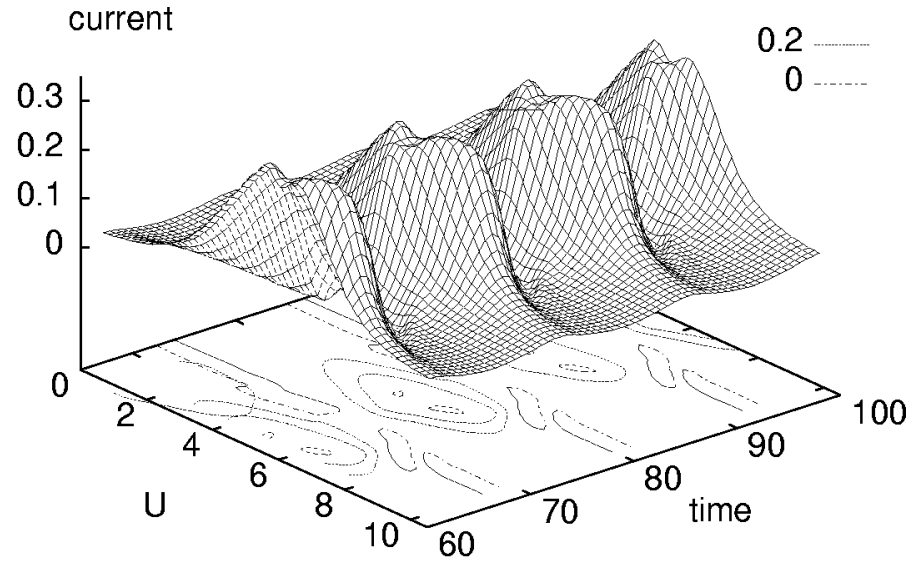

Fig. 6. The current as a function of time and correlation strength $U$ for harmonic modulation with $\omega=0.5, \Delta_{\mathrm{L}}=2, \Delta_{d}=1, \mu_{\mathrm{L}}=2$, and $\varepsilon_{d}=-4$.

to the current curves given on left panels, respectively. In order to give some insight into very complicated structure of the $I_{\mathrm{L}}(\mathrm{t})$ we show in Fig. 8, as an example, the time-dependent current vs. gate voltage for $U=10, \omega=0.5, \Delta_{\mathrm{L}}=2$, and $\Delta_{d}=1$.

As we mention in the introduction, our calculations of the current flowing through the QD and the charge accumulated on it is based on the theory given by Sun and Lin [7] but with one important exception. We were able to calculate in a fully self-consistent manner the QD spin-dependent charge and current flowing in the system. On the contrary, in Ref. [7], this self-consistency was missed and $A_{\alpha}^{\sigma}(\varepsilon, t)$ was calculated replacing the time-dependent QD charge on the right-hand side of Eq. (5) by a constant value. We repeated the calculations (for some parameters) along this way and replaced the QD charge by a constant value (obtained exactly within our approach). The comparison of results of the averaged current vs. gate voltage obtained within our fully self-consistent approach (solid 


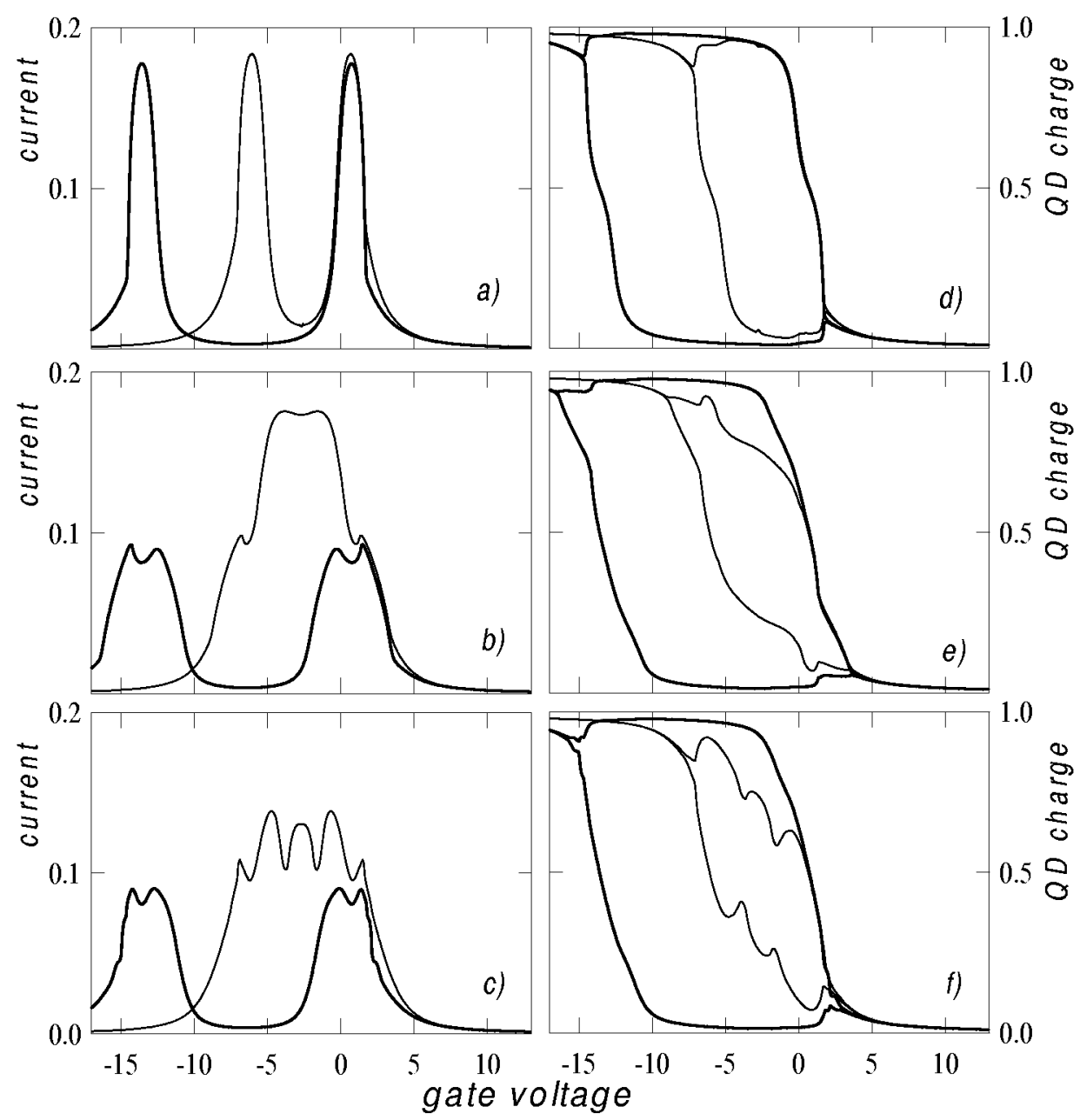

Fig. 7. The average current (left parts) and the quantum dot charge (right parts) as functions of the gate voltage (in $\Gamma$ units) for correlation strength $U=15$ (thick lines) and $U=7.5$ (thin lines). Parts (a) and (d) correspond to a static case and parts (b), (e) and (c), (f) correspond to harmonic modulation frequency $\omega=0.1$ and 0.5 , respectively. $\Delta_{\mathrm{L}}=4, \Delta_{d}=2$, and $\mu_{\mathrm{L}}=2$.

curves) and according to Ref. [7] is given in Fig. 9. These results were obtained for $U=10, \omega=0.5$ and for two oscillation amplitudes $\Delta_{\mathrm{L}}=2$ and $4\left(\Delta_{d}=1\right.$ and 2 , respectively), parts (a) and (b), respectively. The same discrepancies are observed for other values of the parameters.

In summary, we have calculated the current flowing through the QD and the charge accumulated on it in the presence of the time-dependent external fields. The Coulomb intra-dot interaction was taken into account within a mean-field approach. By using the nonequilibrium Green function technique the time-dependent and averaged current and QD charge was calculated in a fully self-consistent way. 


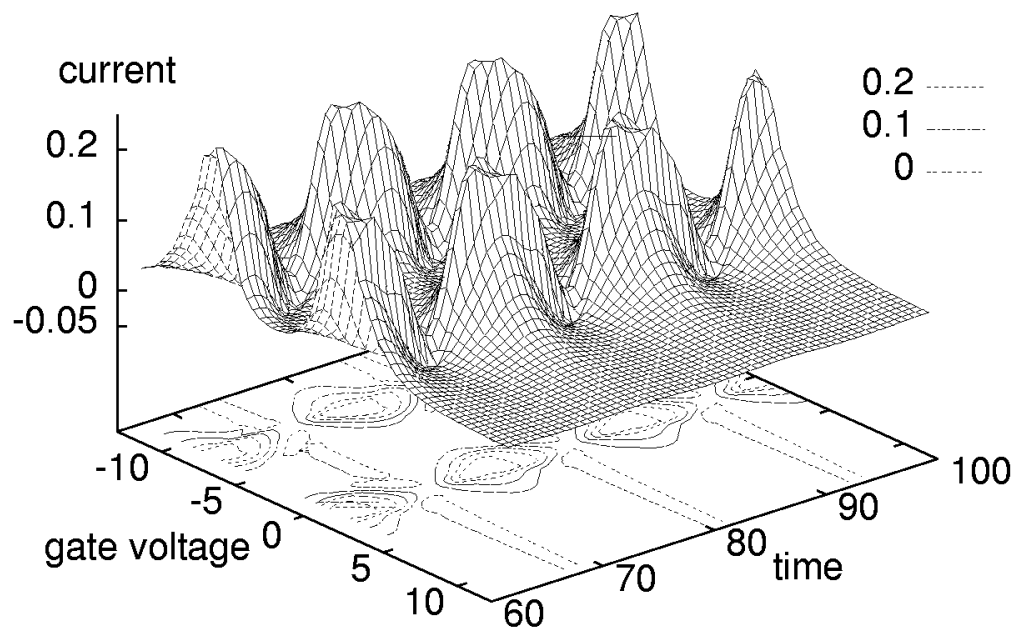

Fig. 8. The current as a function of time and gate voltage (in $\Gamma$ units) for $U=10$, $\Delta_{\mathrm{L}}=2, \Delta_{d}=1, \omega=0.5$, and $\mu_{\mathrm{L}}=2$.

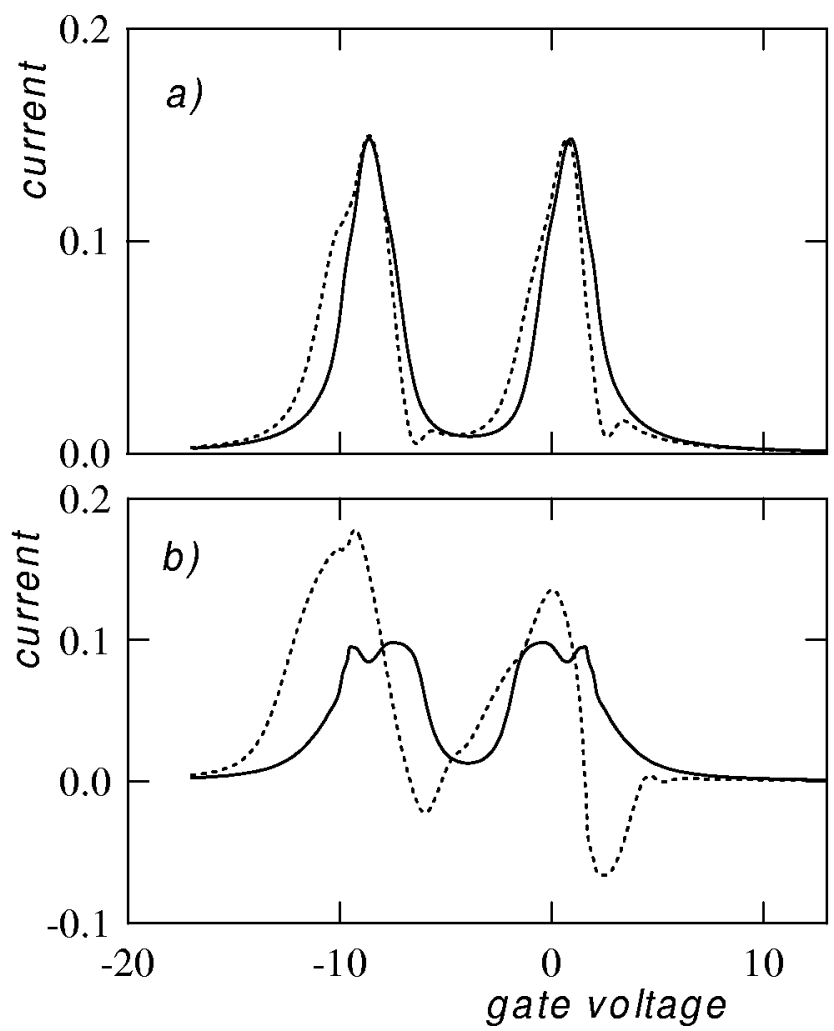

Fig. 9. The average current vs. gate voltage (in $\Gamma$ units) calculated according to Ref. [7] - broken lines and in a fully self-consistent way - solid lines. Parts (a) and (b) correspond to $\left(\Delta_{\mathrm{L}}, \Delta_{d}\right)$ equal to $(2,1)$ and $(4,2)$, respectively. $U=10, \omega=0.5, \mu_{\mathrm{L}}=2$. 
The main conclusions can be summarized as follows:

- With increasing correlation strength $U$ the amplitude of the time current oscillations are damped irrespective of the frequency of external fields (Fig. 2).

- The filling of the spin-polarized QD effective levels is much more asymmetric for greater $U$ (Fig. 3)

- The existence of the spin-polarized solutions for the QD charge results in conductance showing two peaks.

- The splitting of peaks of the differential conductance vs. source-drain voltage curve is observed.

- The average current vs. gate voltage curve has a clear peaked structure for non-oscillating case (Fig. 7a) but this picture is destroyed for nonzero frequencies of the external fields especially for smaller $U$ (Fig. 7c).

\section{References}

[1] L.P. Kouwenhoven, S. Jauher, J. Orenstein, P.L. McEuen, Y. Nagamune, J. Motohisa, H. Sakaki, Phys. Rev. Lett. 73, 3443 (1993).

[2] L.P. Kouwenhoven, A.T. Johnson, N.C. van der Vaart, A. van der Enden, C.J.P.M. Harmans, C.T. Foxon, Z. Phys. B, Condens. Matter 85, 381 (1991).

[3] H.-K. Zhao, J. Wang, Eur. Phys. J. B 9, 513 (1999).

[4] Y. Goldin, Y. Avishai, Phys. Rev. B 61, 16750 (2000).

[5] A.-P. Jauho, N.S. Wingreen, Y. Meir, Phys. Rev. B 50, 5528 (1994).

[6] Qing-feng Sun, Thung-han Lin, Phys, Rev. B 56, 3591 (1997).

[7] Qing-feng Sun, Tsung-han Lin, J. Phys., Condens. Matter 9, 4875 (1998).

[8] Qing-feng Sun, Jian Wang, Tsung-han Lin, Phys. Rev. 58, 13007 (1998).

[9] R.H. Blick, R.J. Haug, D.W. van der Weide, K. von Klitzing, K. Eberl, Appl. Phys. Lett. 67, 3924 (1995).

[10] Qing-feng Sun, Tsung-han Lin, Physica E 4, 201 (1999).

[11] P. Pals, A. MacKinnon, J. Phys., Condens. Matter 8, 5401 (1996).

[12] H. Haug, A.-P. Jauho, Quantum Kinetics in Transport and Optics of Semiconductors, Springer, Heidelberg 1966.

[13] S. Nonoyama, A. Oguri, Y. Asano, S. Maekawa, Phys. Rev. 50, 2667 (1994).

[14] R. Lopez, R. Aguado, G. Platero, C. Tejedor, Phys. Rev. Lett. 81, 4688 (1998).

[15] D. Goldhaber-Gordon, J. Goeres, M.A. Kastner, H. Shtrikman, D. Mahalu, U. Meirav, http://xxx.lanl.gov/abs/cond-mat/9807233 (1998).

[16] S.M. Cronenwett, T.H. Oosterkamp, L.P. Kouwenhoven, Science 281, 540 (1998).

[17] R.H. Blick, R.J. Haug, D.W. van der Weide, K. von Klitzing, K. Eberl, Appl. Phys. Lett. 67, 3924 (1995).

[18] D.M. Newns, Phys. Rev. 178, 1123 (1968).

[19] A. Yoshimori, H. Kawai, M. Makoshi, Progr. Theor. Phys. 80 (Suppl.), 203 (1984).

[20] R. Taranko, E. Taranko, Surf. Sci. 441, 167 (1999). 